\title{
Pubblica utilità: nuovi e vecchi compiti del Design della comunicazione
}

\section{Sommario:}

II progetto WeMi. La città per il welfare costituisce il fulcro di questo contributo poiché si offre come caso paradigmatico per compiere, ex post, alcune riflessioni sul terreno disciplinare del Design della comunicazione e, più specificamente, del Design della comunicazione per il welfare.

Si tratta di riflettere su nuovi e vecchi compiti del Design della comunicazione, sulle responsabilità e funzioni del Design della comunicazione per il Welfare, considerando le sfide progettuali e le Insidie che la trasformazione, guidata dal ruolo assunto dalla Rete e dai media digitali, impone a questo campo del progetto. Ma anche di soffermarsi sul modello di società che si intende promuovere, interrogandosi sui modi più adeguati per favorire spazi di incontro, tra designer della comunicazione e istituzioni pubbliche, sul piano delle comuni responsabilità culturali.

Parole chiave: Design della comunicazione per il Welfare, Sistema di identità e identità dinamica, Responsabilità, Progettazione partecipata

\section{Publicness: new and old tasks of Communication Design}

\section{Abstract}

The WeMi. La città per il welfare project is at the heart of this contribution as it offers a paradigmatic case for carrying out, ex post, some reflections on the disciplinary field of communication design and, more specifically, the design of communication for welfare.

It is an opportunity to reflect on the new and old tasks of Communication Design, on the responsibilities and functions of the Communication Design for Welfare, considering the design challenges and the pitfalls that transformation, guided by the role assumed by the Net and digital media, requires of this field. But also to dwell on the model of society that we intend to promote, questioning ourselves on the most appropriate ways to favor meetings, between communication designers and public institutions, on the level of common cultural responsibilities.

Keywords: Communication Design for Welfare, Identity system and dynamic identity, Responsibility, Participatory design

1 Valeria Bucchetti, professoressa associata, Dipartimento di Design, Politecnico di Milano. valeria.bucchetti@polimi.it 


\section{WeMi. Un progetto per i servizi domiciliari della città di Milano}

Il progetto documentato dal corredo iconografico costituisce il fulcro di questo contributo poiché rappresenta un caso-studio che per finalità, struttura, vocazione, conduzione e durata, ha costituito un banco di prova che si offre come caso paradigmatico per compiere, ex post, alcune riflessioni sul terreno disciplinare del Design della comunicazione e, più specificamente, del Design della comunicazione per il welfare.

Il progetto WeMi. La città per il welfare ${ }^{2}$ è stato realizzato nel quadro delle azioni promosse dal Comune di Milano ed è stato sviluppato da un gruppo interdisciplinare composto da rappresentanti della pubblica amministrazione, operatori sociali, sociologi, architetti ed esperti di studi urbani, esperti di mediazione e designer della comunicazione.

Si è trattato di un progetto complessivo di revisione del sistema dei servizi domiciliari erogati dal Comune di Milano, che si prefissava la costruzione di un sistema di welfare non più legato esclusivamente alla risoluzione dei bisogni primari, ma in grado di diffondere la cosiddetta cultura del benessere della persona. Con queste finalità al progetto di comunicazione è stato attribuito il compito di accompagnare i cittadini e le cittadine, ma anche i diversi attori coinvolti, in questo percorso di trasformazione che ha implicato e implica un cambiamento del punto di vista, dei modi di entrare in contatto con i servizi, di richiederli, di pensarli e condividerli, ossia di una più generale trasformazione dei modelli mentali riferiti alla relazione cittadini-istituzioni. II ruolo del progetto della comunicazione, pertanto, è stato messo in gioco con i propri strumenti, con lo scopo di affiancare le trasformazioni che le politiche sociali stanno compiendo (Rosina \& Sorgi, 2016) e di accompagnare il cambiamento ${ }^{3}$.

In questo suo ruolo di traghettatore il design della comunicazione si è concentrato sulla progettazione di artefatti che rispondono alle necessità comunicative del sistema stesso e che includono differenti funzioni. In primo luogo la funzione identificativa attraverso la progettazione degli elementi di identità visiva del sistema di immagine coordinata che implica, come è noto, la costruzione collettiva del ritratto del soggetto (nel nostro caso, del sistema dei servizi domiciliari del Comune di Milano). A questo scopo sono stati definiti: qualità, aspirazioni, finalità e tratti peculiari, per arrivare a tracciare sia il profilo della figuratività aspettuale di WeMi, sia il profilo dell'articolazione della sua figuratività strutturale (Anceschi,1992, p.19) determinando così presupposti e punti di riferimento utili alla fase progettuale del sistema di immagine coordinata (dallo studio per l'attribuzione del nome, sino alla definizione del sistema di segni in grado di parlare in sua vece).

2 II progetto "WeMi. La città per il welfare" è stato sviluppato nell'ambito del progetto di Fondazione Cariplo "Welfare in azione" (capofila: Comune di Milano, Assessorato Politiche Sociali, Diritti e Salute) 2015-2018. In particolare, il progetto di comunicazione è stato sviluppato dal gruppo DCxW - Design della comunicazione per il Welfare del Dipartimento di Design del Politecnico di Milano (partner del proegtto) formato da: Valeria Bucchetti (responsabile del progetto), Umberto Tolino, Pamela Visconti con Paolo Panzuti Bisanti; al progetto della piattaforma digitale (wemi.milano.it), oltre al gruppo di lavoro, hanno contribuito Giovanni Baule e Marco Quaggiotto. II sistema

3 Di interesse sul tema del bene comune, la prefazione di Stefano Rodotà al volume Del comune o della rivoluzione nel XXI secolo di Pierre Dardot e Christian Laval (2015): https://www.che-fare.com/quale-spazio-per-i-beni-comuni/. 


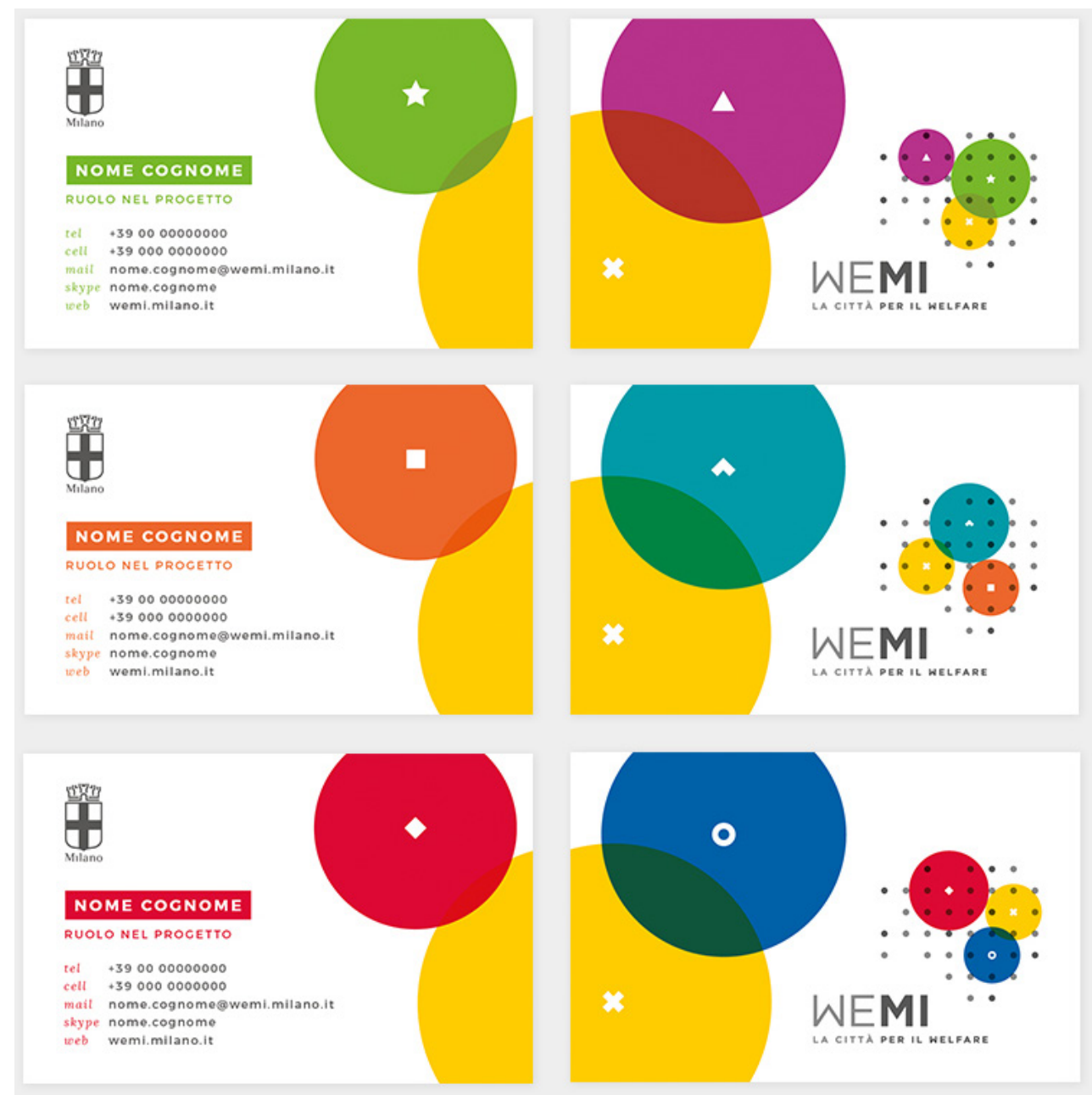

Fig. 1. Alcuni esempi di artefatti. Applicazione delle variazioni grafica ai biglietti da visita.

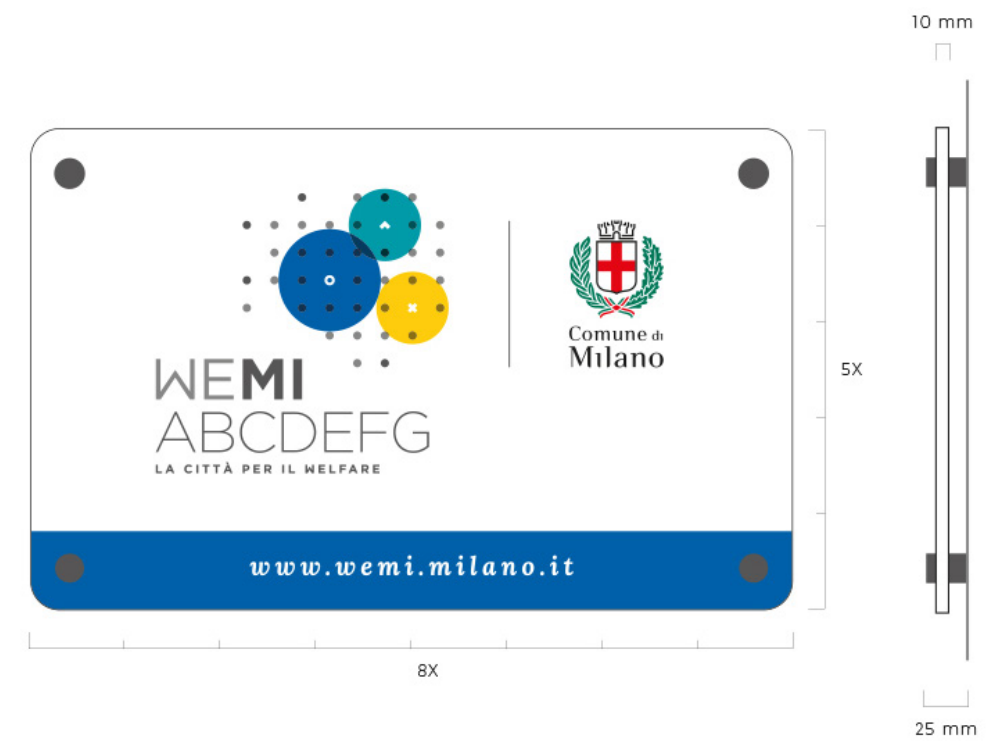

Fig. 2. Elementi di segnaletica. Targa per esterni. 


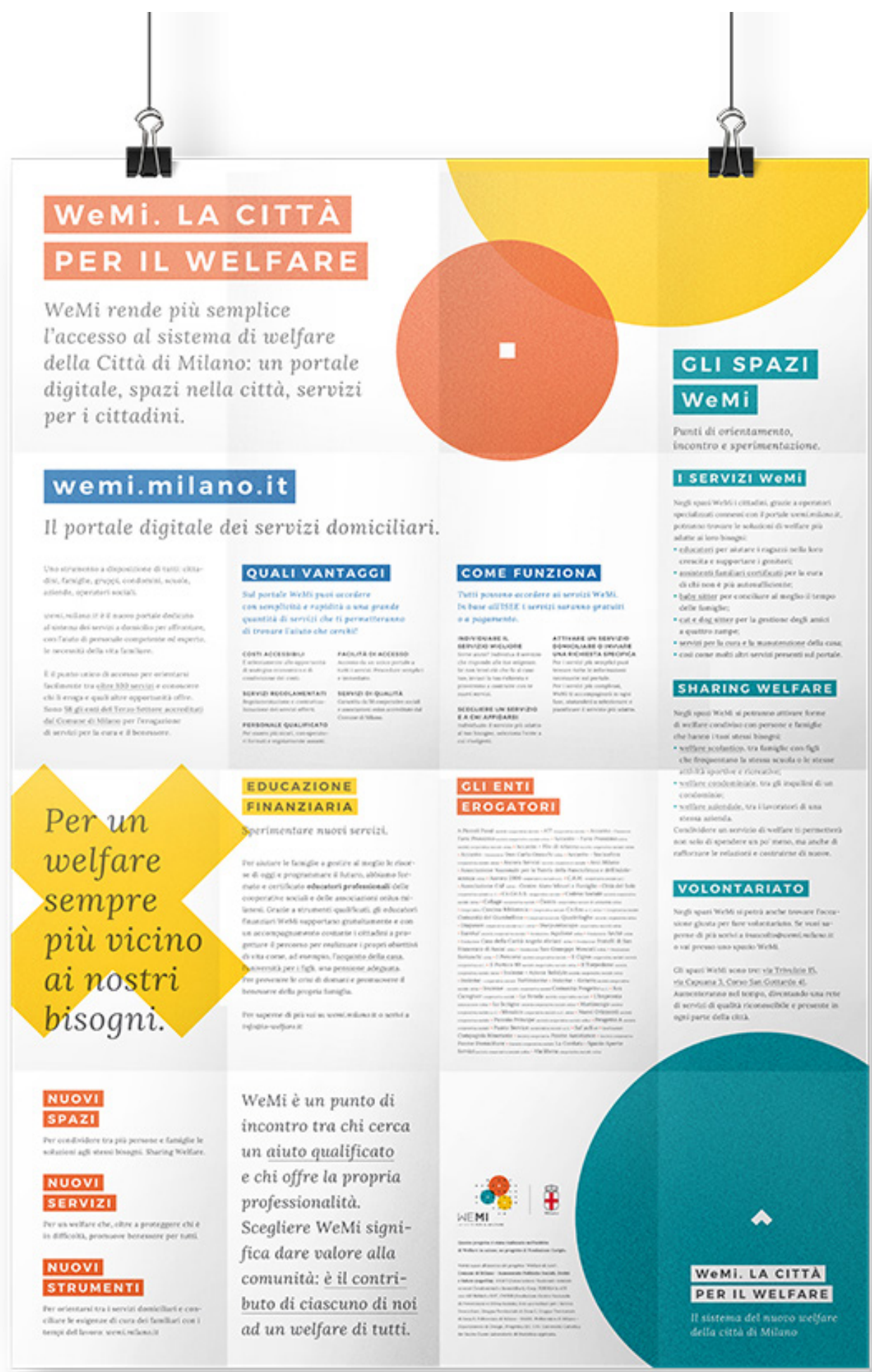

Fig. 3. Manifesto per la presentazione di WeMi e, implicitamente, per il suo sistema di identità. 

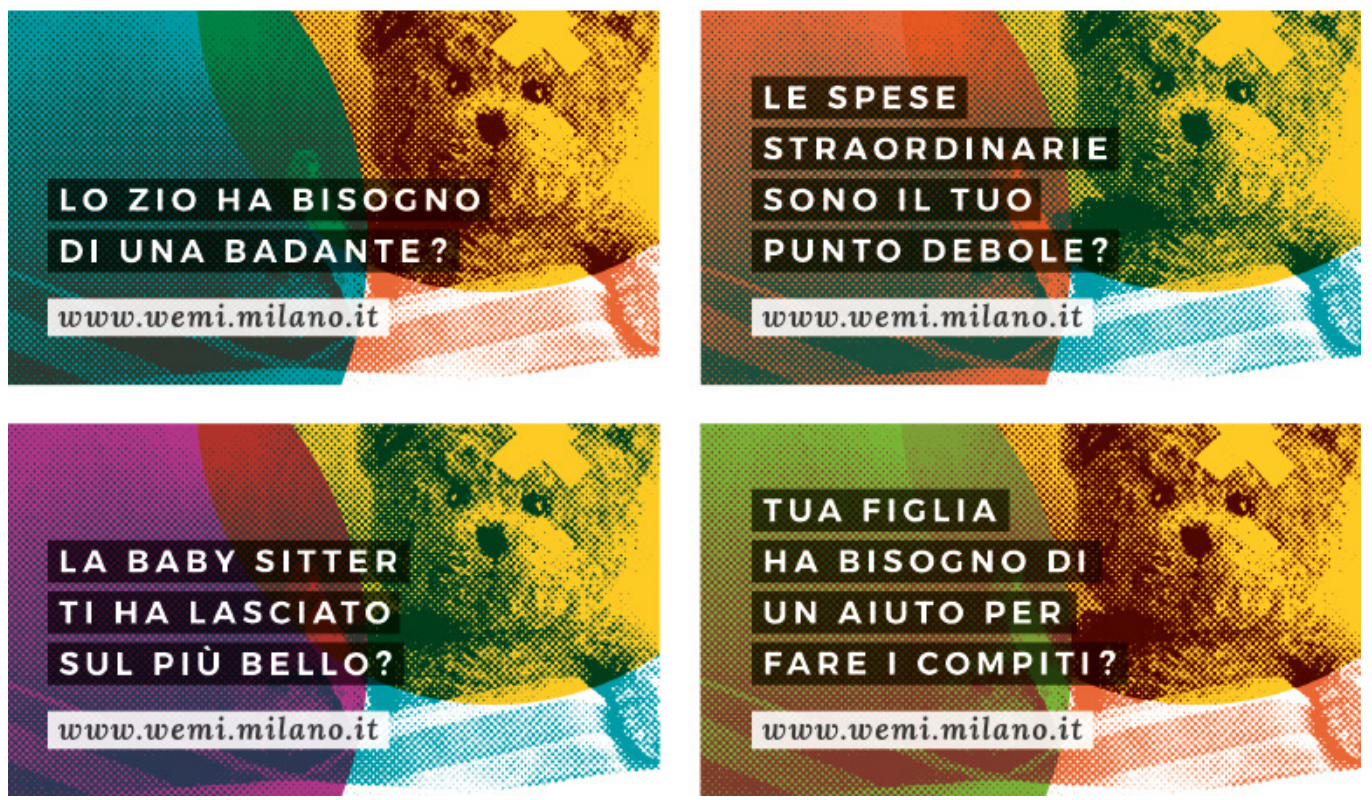

Fig. 4. Flyer dedicati agli spazi WeMi. Declinazioni della copertina

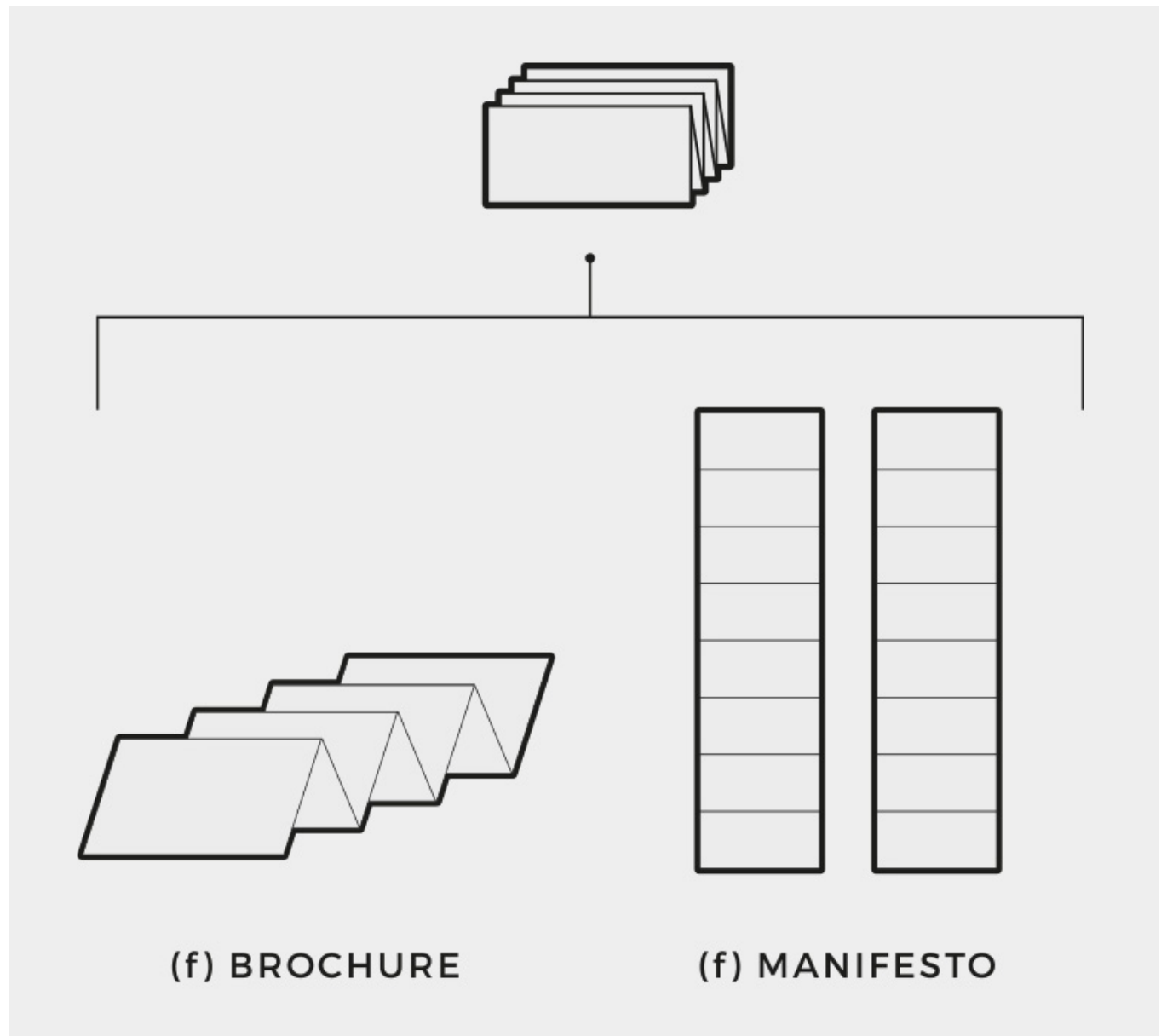

Fig. 5. Flyer dedicati agli spazi WeMi. Struttura dell'artefatto e modalità di fruizione 

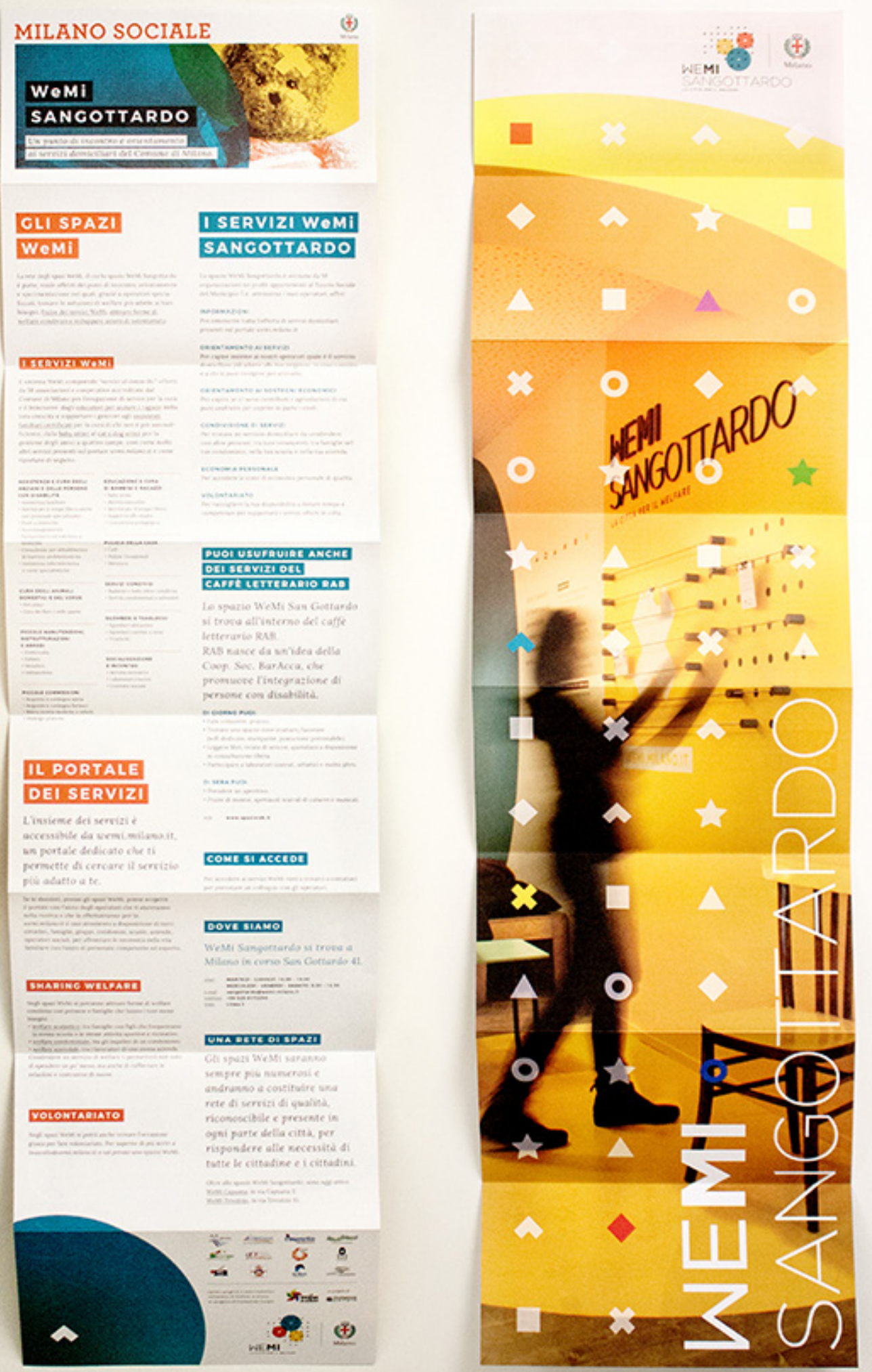

Fig. 6. Manifesto per la presentazione di WeMi, Prototipo 


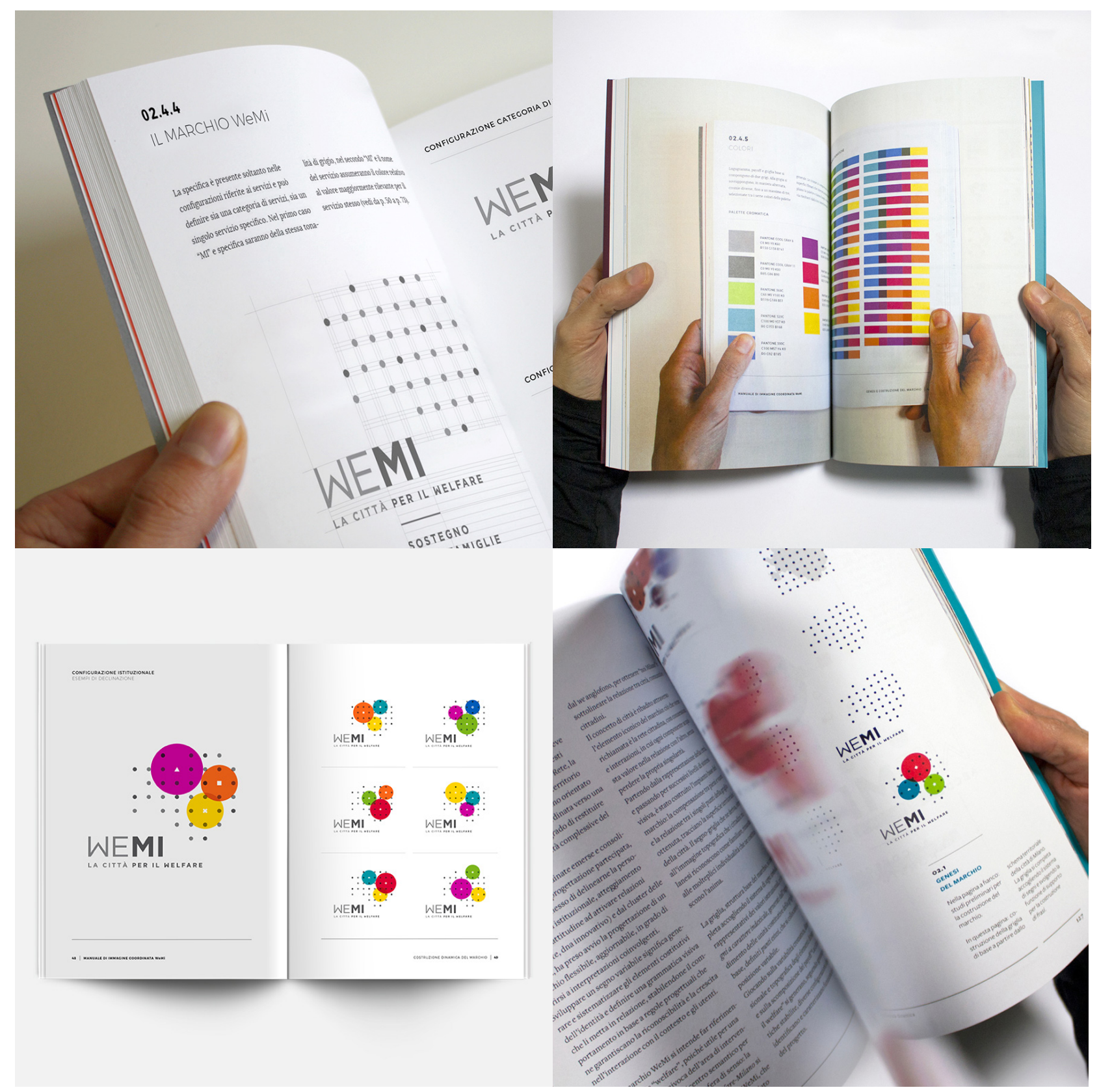

Fig. 7. Alcuni pagine-tipo del manuale di immagine coordinata.

In secondo luogo, la funzione informativa-promozionale, che prevede la progettazione di strumenti comunicativi e allestitivi in grado di veicolare informazioni sul sistema per diffonderne contenuti e peculiarità; funziwone che è stata esercitata attraverso la progettazione degli elementi primari del sistema di immagine coordinata e, in particolare, di una piattaforma digitale per l'accesso ai servizi domiciliari (portale in Rete - wemi.milano. it), ma anche di un apparato comunicativo per gli spazi WeMi, ossia luoghi fisici (piattaforme territoriali) che costituiscono spazi di incontro, punti di contatto e di scambio con i cittadini e le cittadine, previsti dalle linee strategiche del progetto. Accanto a esse, la funzione fàtica; messa in atto tramite un insieme di strumenti idonei ad attivare e rinnovare le relazioni, a mantenere vivi i canali di scambio con i cittadini e a favorire modalità dirette e indirette di ascolto. Sino ad arrivare al piano sistemico che investe la definizione di regole 
e grammatiche per la gestione e la tenuta di tipo registico degli artefatti, affinché la produzione si mantenga nel tempo coerente nella sua articolazione, aderente al soggetto, e a esso riconducibile. A questo piano è destinata la progettazione di due dispositivi: uno più canonico - il manual - e un supporto digitale - l'identity toolkit - per la generazione del marchio dinamico.

Ai compiti che sono tradizionalmente di stretta pertinenza del progetto della comunicazione, e che danno forma all'interfaccia comunicativa tra soggetto e cittadinanza, se ne è affiancato un altro. II Design della comunicazione, in questo quadro, ha portato il proprio contributo a livelli molteplici, attivando ambiti delle discipline del progetto che hanno assunto pesi e funzioni via via differenti nel corso delle singole fasi del processo progettuale.

Alla figura del designer della comunicazione è stata infatti affidata anche una funzione di "accompagnamento" dell'intero processo, includendo il suo punto di vista disciplinare nel corso dello sviluppo delle azioni, così da alimentare il confronto con gli altri soggetti coinvolti nella definizione del sistema dei servizi domiciliari e di consentire, durante il processo decisionale, l'arricchimento del dialogo tra le parti.

Grazie a questo ruolo le azioni comunicative non sono state previste a valle, come frequentemente accade, ma maturate in itinere attraverso un lavoro di traduzione costante delle diverse istanze, accompagnando cioè i processi decisionali tramite un percorso che facilita e rende maggiormente afferrabile, grazie alla loro forma visibile, le implicazioni che gli scenari e le ipotesi delineate avrebbero comportato. È stata messa in atto quella capacità del designer di inquadrare e guidare la propria ricerca e di orientarla agli accadimenti auspicati portandola nel futuro anteriore (Celaschi, 2016, p.49-50). Gli strumenti progettati dal designer della comunicazione sono stati messi, a questo scopo, al servizio dell'intero gruppo per dare forma visuale al sistema in fieri, facilitando decisioni e processi di anticipazione o di previsione strategica.

Si tratta di un ruolo di grande rilevanza, in modo particolare quando si ragiona su processi di modificazione della realtà che riguardano la dimensione strategica della pubblica amministrazione, delle imprese, dei territori, di grandi progetti integrati. Con il progetto WeMi ci si è, infatti, trovati di fronte a una rete di soggetti appartenenti all'amministrazione locale, all'università, unitamente a una cordata di organizzazioni del terzo settore (cooperative, imprese sociali ecc.), che hanno lavorato congiuntamente per la costruzione di un modello di welfare volto a superare la frammentazione dell'offerta dei servizi domiciliari, ad abbattere le barriere tra $\mathrm{i}$ cittadini e a favorire la creazione di valore sociale e legami interpersonali.

Il compito del designer, in questo contesto, è stato dunque quello di «stare in squadra con altri demiurghi che svolgono parti del processo altrettanto imponenti e importanti: collaborare, integrare le proprie competenze, partecipando così a un sistema di lavoro articolato, temporalmente importante, costellato di portatori di interesse e pieno di incertezze decisionali». (Celaschi, 2016, p.50). Il design della comunicazione ha, pertanto, esteso il proprio ruolo di traduttore tra emittente e destinatario (Anceschi, 1981; Baule \& Caratti, 2016) per rivestire contemporaneamente quello di attore all'interno di un sistema di soggetti e di facilitatore delle relazioni all'interno del gruppo di lavoro.

\section{Nuovi e vecchi compiti del Design della comunicazione}

In questa prospettiva è possibile parlare di Design della comunicazione per il welfare. Con questa espressione non si intende tanto costituire una delimitazione di campo, ma esplicitare la specificità di un'area di intervento per portare l'attenzione sulla sua centralità e sulle competenze disciplinari necessarie, che ne sono implicate e che ne determinano l'agito. 
Ciò significa rimettere il focus su questioni che riguardano il rapporto tra progetto della comunicazione e società e, implicitamente, ribadire l'urgenza di riaccendere un dibattito sui temi che questa relazione sottende, per ridefinirne obiettivi e priorità. Parlare di design della comunicazione per il welfare vuol dire anche ribadire una prospettiva di responsabilità che, sebbene debba essere un prerequisito della comunicazione tout court, si fa tratto imprescindibile nel momento in cui coinvolge ciò che appartiene tradizionalmente alla progettazione di pubblica utilità o grafica di utilità sociale (Anceschi, 1984; Pignotti, 1984). Un'area del progetto, questa, che ha radici profonde e strade tracciate (Steiner, 1973) e che, seppure con linee di lavoro differenti, ha visto periodicamente riaffermare l'idea di un "mestiere" di progettista della comunicazione aperto al dialogo, alle tensioni della società, capace di sostenere un'alternativa all'ideologia della professionalizzazione, sino a richiamare il progettista ad assumere un coraggio "etico" e a riconsiderare obiettivi e contenuti nei quali riversare il proprio "talento".

Siamo di fronte a un asse forte che, seppure minato nel corso del tempo da derive omologanti e commerciali e da stereotipi espressivi ${ }^{5}$, ha permesso di tracciare una traiettoria netta: dalla grafica di pubblica utilità - quando la distinzione tra pubblico e privato era chiara e inequivocabile e quando il progetto aveva le caratteristiche proprie dell'era pre-digitale -, verso una sua trasformazione che intende mantenere inalterato il valore sociale della comunicazione. II percorso intrapreso, e nel quale siamo ancora immersi, ha incluso quei caratteri evolutivi che ne hanno progressivamente ridisegnato il campo, permettendo di accogliere i diversi accenti. Si intersecano Design della comunicazione per la responsabilità sociale, Design dell'accesso (Baule, 2012), Social design, Design per la comunicazione sociale (Galbiati \& Piredda, 2012), Design for social innovation and sustainability ${ }^{6}$ con i loro valori, ossia ambiti accomunati dalle nozioni di etica, sostenibilità, accessibilità, partecipazione: principi comuni e portanti per ciascuno di essi ${ }^{7}$.

La funzione di servizio rivolta alla collettività viene condivisa e la persona è posta al centro. Un oggetto individuabile nell'interesse generale prevale - secondo l'accezione di Design for Human Scale di Papanek $(1971 ; 1983)$ e quanto suggerito dalla relazione tra democrazia e design promossa da Manzini e Margolin ${ }^{8}$-e impone una prospettiva vigile e una volontà di azione volta a far emergere all'attenzione pubblica voci e temi riferiti a questioni non adeguatamente considerati dai media mainstream.

Ci confrontiamo, cioè, con un ambito sfaccettato in cui, per dirla con Mancini, i due termini public affairs e

\footnotetext{
4 Come sostiene Papanek (1995) i progettisti hanno la possibilità di fare qualche cosa di nuovo o di riqualificare qualche cosa in modo che diventi migliore, ponendo in evidenza potenzialità e responsabilità dell'agire progettuale. Sul tema dell'altra grafica, ossia quella che si oppone alla grafica di consumo, si veda la voce Altra grafica ( $L^{\prime}$ ) redatta da Mario Piazza nel catalogo Annisettanta (Belpoliti, Canova, Chiodi 2007); sul tema dell'etica cfr. Adriano Fabris (2006); No Brand more profit / Etica e comunicazione (Bollini \& Branzaglia, 2003) in particolare il saggio di Letizia Bollini dedicato ai "Manifesti" come coscienza etica della professione.

5 Si pensi alle azioni di richiamo esercitate nei confronti dei messaggi discriminatori della pubblicità; tra queste: la Risoluzione del Parlamento europeo del 3 settembre 2008 sull'impatto del marketing e della pubblicità sulla parità tra donne e uomini (2008/2038(INI)); la Risoluzione del Parlamento europeo dell'8 marzo 2011; o alla stesura di documenti come la Carta di Milano. Per il rispetto delle bambine e dei bambini nella comunicazione promossa da Terre des hommes (http://www.cartadimilano.org) contro l'uso dell'immagine di bambine e bambini quando si presta a un impiego strumentale che ne sminuisce la dignità e rafforza stereotipi discriminatori o costruire stili di vita pericolosi.

6 Si veda il lavoro di Desis Lab. dedicato allo sviluppo di ricerche sul Design per l'innovazione sociale e la sostenibilità, la progettazione strategica e dei servizi, i metodi e gli strumenti di co-design (Politecnico di Milano).

7 Per un allargamento della riflessione si rimanda al contributo di Giovanni Lussu e alle posizioni espresse durante la conferenza Grafica e politica. Grafica è politica. Indizi (maggio 2017, ISIA, Urbino).

8 Si fa riferimento alla relazione tra democrazia e design promossa attraverso la lettera aperta "Stand up for Democracy" di Ezio Manzini e Victor Margolin (2017).
} 
publicness assumono un ruolo cardine. II primo esprimendo gli affari che riguardano l'intera comunità, anche se non sempre di facile individuazione, il secondo facendo riferimento a una sorta di proprietà delle istituzioni e dei loro argomenti, ossia, essere accessibili, aperte al pubblico e disponibili a fornire informazioni di interesse generale (Mancini, 2002a; 2002b). Ed è in questo quadro che si colloca il progetto WeMi; le sue azioni comunicative riguardano, infatti, un'offerta il cui campo di pertinenza è delineato da funzioni socialmente rilevanti al servizio delle politiche sociali (Mancini, 2002b, p.7): quelle che hanno a che fare con il benessere e le condizioni di vita delle persone, con le risorse e le opportunità a loro disposizione nelle varie fasi della loro esistenza, ossia aree di progetto determinanti per il miglioramento della qualità della vita. Aree all'interno delle quali ridisegnare nuove priorità nella scala dei valori progettuali ${ }^{9} \mathrm{e}$, perché no, anche professionali; in equilibrio tra valori umanistici e conoscenza tecnica, da impiegare entrambi in un attento e cosciente processo critico che abbia come scopo il miglioramento delle condizioni di vita sociali e ambientali (Bollini, 2003, p. 54).

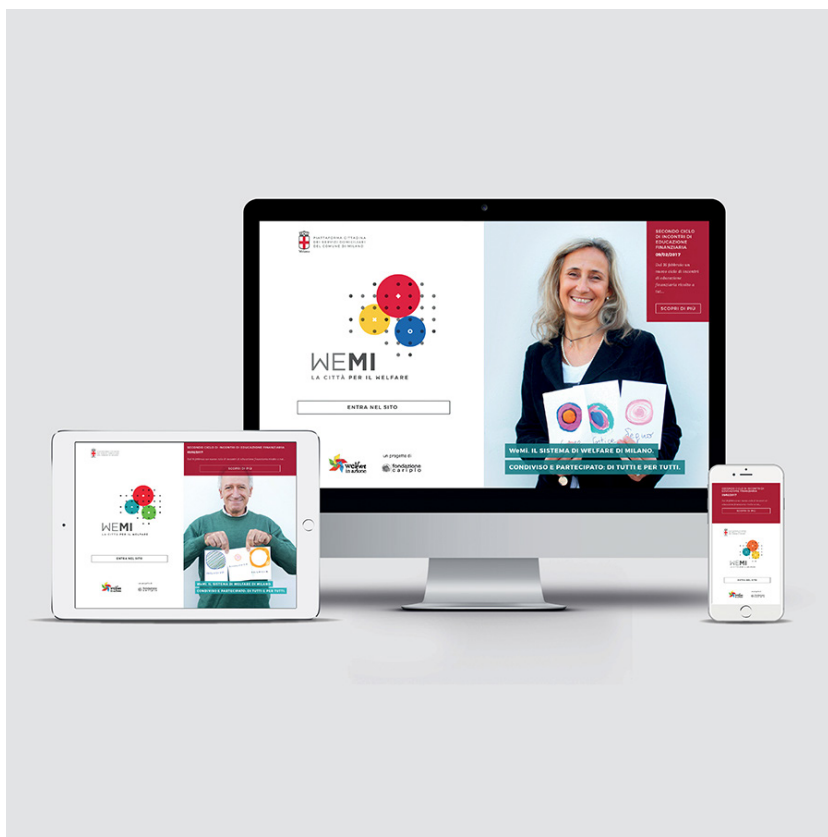

Fig. 8. Schermata di ingresso al portale wemi.milano.it La pagina di benvenuto introduce la galleria dei testimoni del sistema che affiancano il marchio nella sua versione cinetica.

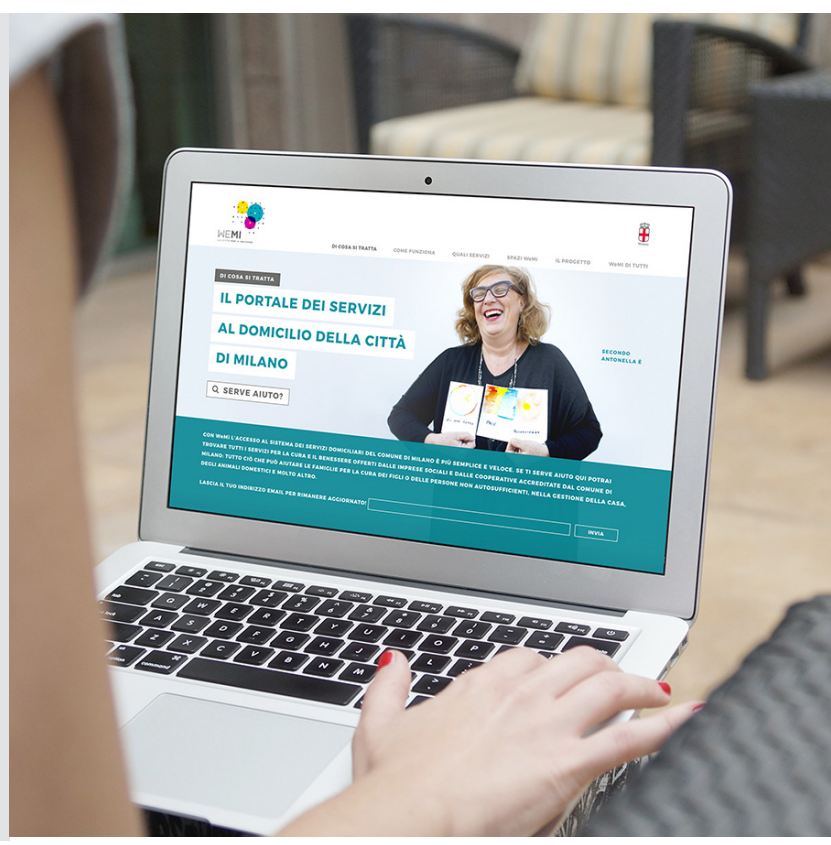

Fig. 9. Pagina di presentazione del servizio.

\section{Responsabilità e funzioni del Design della comunicazione per il Welfare}

Secondo questi presupposti, ha preso avvio il lavoro dei progettisti della comunicazione che, di fronte alle sfide che il sistema WeMi ha imposto di affrontare, hanno messo in atto un comportamento da soggetti che agiscono nella collettività per la collettività, assumendo la responsabilità dei segni e delle tracce che lasciano nella semiosfera, della qualità dell'atto comunicativo stesso, al quale è strettamente connessa la qualità dell'accesso ai contenuti ${ }^{10}$.

9 Di particolare interesse la ricognizione sulla comunicazione pubblica condotta da Margherita Ider alla base della sua tesi di laurea La comunicazione come servizio pubblico. Teorie e prassi del design della comunicazione per il sociale (a.a. 2012-2013, Corso di laurea Magistrale in Design della comunicazione, Scuola del Design, Politecnico di Milano, relatore prof. Valeria Bucchetti).

10 Cfr. il numero 22 della rivista Progetto grafico dedicato al tema Spazio comune. 
Si tratta di atti comunicativi che vengono articolati in funzione di compiti specifici; da compiti che possiamo definire di servizio, che implicano quel versante di "ingegneria della comunicazione", che agisce laddove è necessario agevolare i processi comunicativi comunitari dell'intera macchina sociale, e l'ambito dell'ergonomia delle comunicazioni sociali e del design dell'accesso (destinati a rendere noto e a consentire il fare). Si pensi, in questo senso, alle forme di comunicazione prescrittiva, a tutte le forme di istruzioni per l'uso che indirizzano e accompagnano i nostri percorsi e le nostre azioni sul territorio per consentirci di raggiungere un luogo, un servizio, un risultato. A compiti di tipo educativo-informativo, caratterizzati da azioni di sensibilizzazione e di formazione delle coscienze e dei comportamenti che, per quanto concerne il contenuto, svolgono una funzione sociale fondamentale «quella di <in-formare>, nel senso di creare una vera coscienza politica, di contrapporsi alla disinformazione dei messaggi pubblicitari e all'informazione parziale, cioè <di parte > di quelli politici, di divulgare e di far conoscere tutto quello che è di interesse per la collettività» ${ }^{11}$. Un compito educativo che vuole favorire dei cambiamenti nell'immaginario, nella sensibilità e nei comportamenti, che può sottendere sia istituzioni pubbliche, sia ambiti privati o aggregazioni no-profit e che si concretizza attraverso comunicazioni mirate a informare la popolazione riguardo specifici problemi sociali, a informare sulla possibilità di adottare un comportamento per prevenire o risolvere un problema (rivolta a promuovere un cambiamento di opinione).

Infine, una terza categoria riguarda i compiti la cui finalità generativa è improntata alla produzione e diffusione di relazioni e di legami sociali (una delle missioni del progetto WeMi), che si collegano alla capacità della comunicazione sociale (Mancini, 2002) di produrre relazioni, solidarietà, tessuto sociale, di svolgere azioni di advocacy ${ }^{12}$. Si tratta in questo caso di azioni comunicative volte a incoraggiare la relazionalità diffusa, la solidarietà e l'inclusione; a incrementare la partecipazione della cittadinanza attiva e la responsabilità sociale nel suo complesso (rivolte a promuovere attraverso l'azione un cambiamento nel modello e nel comportamento) e che per essere agite richiedono la partecipazione allargata della società civile, l'impulso di molteplici attori che promuovano idee, progetti, servizi di interesse pubblico.

\section{Insidie e sfide progettuali}

La fase di progettazione del sistema comunicativo di WeMi e la progettazione degli artefatti on e offline hanno riportato all'attenzione alcune questioni, non certo nuove, ma che ancora oggi necessiterebbero di essere affrontate in profondità, non solo dalle discipline del progetto, quant'anche in un quadro di confronto interdisciplinare.

Il lavoro compiuto nell'area del design della comunicazione per il welfare ${ }^{13}$ ha offerto cioè un terreno di sperimentazione, ma anche un'opportunità per interrogarsi nel merito, su alcuni temi che di seguito mi limiterò a enunciare.

\footnotetext{
11 È quanto riporta Lamberto Pignotti facendo riferimento a uno studio di Lidia Leo e alle sue affermazioni. Si veda Pignotti (1984).

Con il termine advocacy si indica l'insieme di azioni con cui un soggetto si fa promotore e sostiene attivamente la causa di un altro.

3 Il gruppo di ricerca di Design della comunicazione del Dipartimento di Design (Politecnico di Milano) da numerosi anni si dedica ai temi della responsabilità sociale portando queste istanze nella formazione e dedicando ai temi del welfare sperimentazioni didattiche (si pensi per esempio ai Laboratori di sintesi DCxW dedicati all'affido familiare, all'omofobia, all'integrazione, alla socializzazione che hanno visto nel corso del tempo il coinvolgimento di Erik Ciravegna, Chiara Diana, Umberto Tolino, Pamela Visconti, oltre a chi scrive con responsabilità di coordinamento.
} 


\section{Quale modello di società}

Una delle questioni imprescindibili si sviluppa attorno alla relazione tra azione comunicativa - sua concretizzazione attraverso dispositivi e formati della comunicazione - e modello di società che attraverso di essa si intende promuovere. Questione che pone il progetto in una prospettiva di comunicazione sostenibile. Come sottolinea Baule (2005, p.12): «ora che l'orizzonte si allarga alle conseguenze non immediate, agli effetti collaterali del nostro agire progettuale, sappiamo che ogni artefatto lascia una traccia, implica l'utilizzo di risorse, occupa uno spazio di attenzione a scapito di un altro, produce scorie. L'etica della responsabilità apre il capitolo di una comunicazione sostenibile, dove conta anche quale modello di società promuove, a quale modello di mondo partecipa». E dunque, anche per quanto concerne il progetto di comunicazione WeMi, il tema del modello di mondo al quale si intende partecipare non poteva essere messo in secondo piano (cfr. Anceschi, 2011).

Le mosse comunicative si sono sviluppate a partire dall'intenzione di pensare a nuove forme di relazione tra cittadini e cittadine, servizi e istituzioni. Da parte del Comune, soggetto responsabile del sistema dei servizi domiciliari, la volontà dunque di proporsi rigoroso e affidabile, ma al contempo innovativo, flessibile, inclusivo; capace di accogliere le esigenze dei singoli e di metterle al centro di un sistema del quale tutti fanno parte. II progetto ha preso dunque forma nella consapevolezza del suo ruolo di traduttore di valori e intenzioni, assumendo si di sé la responsabilità della creazione di un apparato utile a veicolarli. L'interfaccia comunicativa che collega contenuti (fatti, servizi, modi di erogarli) e destinatari partecipa così alla costruzione della percezione del sistema. Alla base della sua progettazione vi è la consapevolezza di essere complici di una costruzione che può essere realistica o distorcente, maschera che può svelare, deformare, promettere, illudere, e che per questo ci richiede di essere sempre vigili rispetto al pericolo di derive populiste che si nutrono di mezzi di comunicazione estetici (Baule, 2017).

\section{La qualità dei segni}

Il piano del linguaggio, il piano dei registri espressivi, rappresenta un secondo tema nodale ricco di sfaccettature e ombre.

Sebbene in passato il modello di espressività standardizzata del linguaggio grafico fu messo in discussione ${ }^{14}$ reclamando uno spazio all'autore, in primo luogo come intellettuale, oggi, a distanza di decenni, fatichiamo ancora a sviluppare un fare progettuale che ne mantenga "alte" caratteristiche e qualità. Ci muoviamo infatti all'interno di un perimetro in cui esercitano la loro influenza, da un lato, il linguaggio dominante della pubblicità commerciale, che si è diffuso in contesti e su scale differenti ${ }^{15} \mathrm{e}$, dall'altro, la cospicua produzione grafica che è spesso frutto di improvvisazione (Visconti, 2017, p.76), favorita dalla diffusione di dispositivi produttivi (software per la gestione del progetto grafico) destinati a utenti generici, a non "addetti ai lavori", che attraverso di essi

\footnotetext{
14 Si fa riferimento alle azioni condotte in particolare negli anni Ottanta che diedero luogo a quello che fu definito un 'movimento progettuale', movimento che nel corso del medesimo decennio perse la propria coesione e parte della propria energia.

15 Si pensi, per esempio, a come la nozione di branding si sia espansa raggiungendo ambiti (come, a titolo esemplificativo, quello territoriale) che le erano estranei dando luogo a forme di comunicazione fortemente ibridate con i linguaggi della pubblicità commerciale.
} 
possono trovare soluzioni grafiche basate sulla semplificazione, la standardizzazione, la banalizzazione ${ }^{16}$.

In modo particolare, possiamo osservare come siano marcati gli effetti della diffusione generalizzata degli strumenti dedicati alla produzione di artefatti grafici e alla loro manipolazione (software per il progetto grafico). E questo è ancora più evidente se consideriamo la produzione della comunicazione di pubblica utilità da parte di istituzioni o di enti (realtà del terzo settore, cooperative sociali ecc.) che rispondono a esigenze di produzioni low-budget e, a volte, a una mancanza di programmazione dell'azione che induce a una progettazione estemporanea accompagnata, nella maggior parte dei casi, da una scarsa cultura visiva da parte di chi agisce il progetto e di chi ha ruoli decisionali in merito a esso' ${ }^{17}$.

Ci troviamo cioè ancora una volta di fronte a quei linguaggi che negli anni Ottanta venivano denunciati come linguaggi soliti far «ricorso al preesistente, al proverbiale, al confezionato, al pronto per l'uso, al luogo comune, al già sentito, al già visto. [...] all'esibizione ripetuta di un limitato repertorio di significanti in galoppante obsolescenza» (Pignotti, 1984, p.36) e il fatto che ancora oggi queste considerazioni trovino riscontro nella realtà ci aiuta a comprendere quanto lontano sia il traguardo.

Non ultimo, va considerato il ruolo sotteso dalle istituzioni pubbliche e ribadita la convinzione che, quando sono loro a parlare, l'impegno a tenere alto il registro linguistico e la qualità estetica del messaggio di cui si fanno portavoce debba essere un implicito, un requisito imprescindibile, unitamente alla loro responsabilità nei confronti di coloro ai quali si parla, espressa secondo principi di inclusione e di equità.

\section{Stabilità temporale e consunzione delle immagini}

Un terzo aspetto riguarda la "fragilità" delle immagini, che la contemporaneità consuma con un ritmo incalzante, una fragilità rafforzata dalla pressione prodotta su di esse dalla dimensione effimera dell'atto comunicativo, dalle forme di spettacolarizzazione capaci di trasformare ogni cosa in "evento", dalla violenza della visibilità (Mondzain, 2015) in un contesto in forte trasformazione guidato dal ruolo assunto dalla Rete e dai media digitali, che con passi assai rapidi stanno orientando il progetto di comunicazione secondo nuovi formati e linguaggi (Sinni, 2018).

In questo quadro, il progetto $\mathrm{WeMi}^{18}$ ha offerto un'opportunità per riflettere sul sistema di identità, sul ruolo del segno-marchio, sulla sua stabilità, ma anche sulle potenzialità del suo essere immagine dinamica capace di costruire consenso e partecipazione, secondo il principio che vede la base del design trasformarsi «da produzione di forme da parte dei professionisti in produzione di azioni da parte dei destinatari» ${ }^{19}$.

Per il sistema di identità WeMi si è scelto pertanto di accettare la sfida e cogliere la dimensione evolutiva come

\footnotetext{
16 Vedi la voce “Design della comunicazione” redatta da Giovanni Lussu, per "treccani.it”, http://www.treccani.it/enciclopedia/(consultato il 25 novembre 2018).

17 La gestione complessa interna alle pubbliche amministrazioni, che riguarda le modalità di conferimento degli incarichi nel rispetto della trasparenza, unitamente alla riduzione di risorse economiche, produce un terreno sensibile alle sollecitazioni esterne, aperto all'attivazione di azioni progettuali che imprese, privati, cittadini si prestano a offrire alle pubbliche amministrazione, per meglio dire, a donare in forma di gratuità e che non necessariamente sono portatrici di qualità.

18 Un'occasione di riflessione che non nasce unicamente dalla sperimentazione condotta durante la costruzione del processo, o nelle fasi di co-progettazione, ma scaturisce anche a partire dalle implicazioni che la progettazione del sistema di identità comporta.

19 Si fa riferimento al manifesto redatto dallo studio newyorchese Project Projects sul Graphic design (in particolare al punto 7), pubblicato sul n. 22. della rivista Progetto Grafico.
} 
fattore caratterizzante, progettando un sistema di identità dinamico predisposto al cambiamento, composto da variazioni, da declinazioni molteplici, da combinazioni di elementi rese possibili da una grammatica e da un abaco di segni-base, ideati per essere affiancati e sovrapposti al fine di costruire segni-marchio adatti a definire i diversi ambiti di azione.

Si è arrivati così a ottenere un sistema di tipo trasformativo, in grado di accogliere ogni nuovo soggetto entri a far parte della rete, dotandolo di un proprio apparato comunicativo conforme al sistema e calato nella propria specifica realtà; un sistema che deve mettere alla prova la propria capacità di ricombinarsi con forme espressive via via distinte, con contesti eterogenei, di accogliere la contaminazione e la forma ibrida come parte costitutiva del suo essere (Bucchetti, 2017; Consalez, 2017).

Sono state cioè compiute scelte progettuali che accolgono la sfida circa la tenuta stessa del sistema, che viene verificata ogni volta in cui esso viene affidato a chi lo vive, lo mette in azione, lo fa proprio, accettando che ogni soggetto possa arricchirlo con la propria "intonazione" e che queste imperfezioni possano essere riassorbite dalla sua stessa capacità di coesione.

Quanto esposto in queste pagine ci invita, pertanto, a rimettere al centro del dibattito i nodi descritti e a mantenere alta la soglia di attenzione affinché il design della comunicazione per il welfare non rinunci a perseguire quel condensato di "alta qualità della cosa pubblica" che deve restare un requisito imprescindibile degli artefatti grafici, una qualità che non coinvolge unicamente la soluzione formale, compositiva, l'estetica della figura, ma anche il contenuto della rappresentazione e che deve imporre ai designer e alle istituzioni pubbliche di incontrarsi sul piano delle comuni responsabilità culturali.
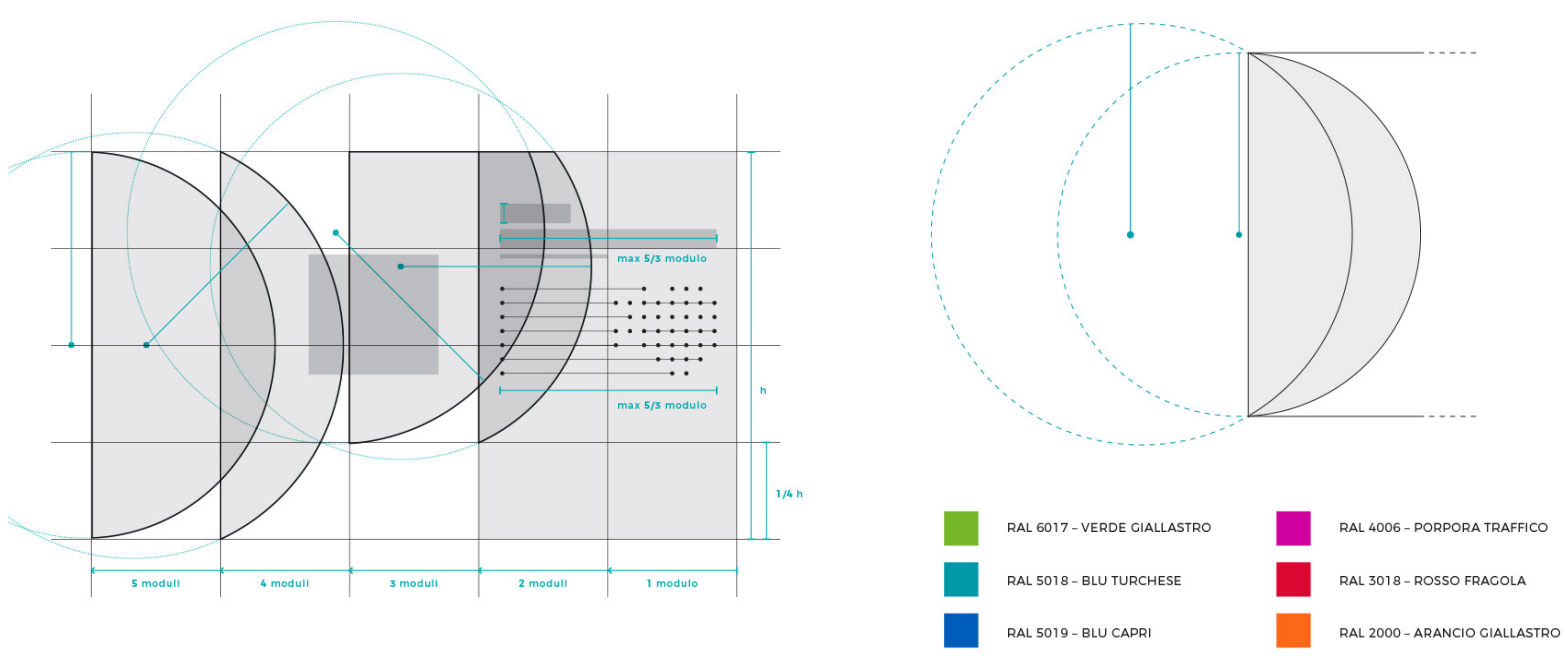

Fig. 10. Costruzione modulare della parete, riferimenti cromatici e dimensionali. 


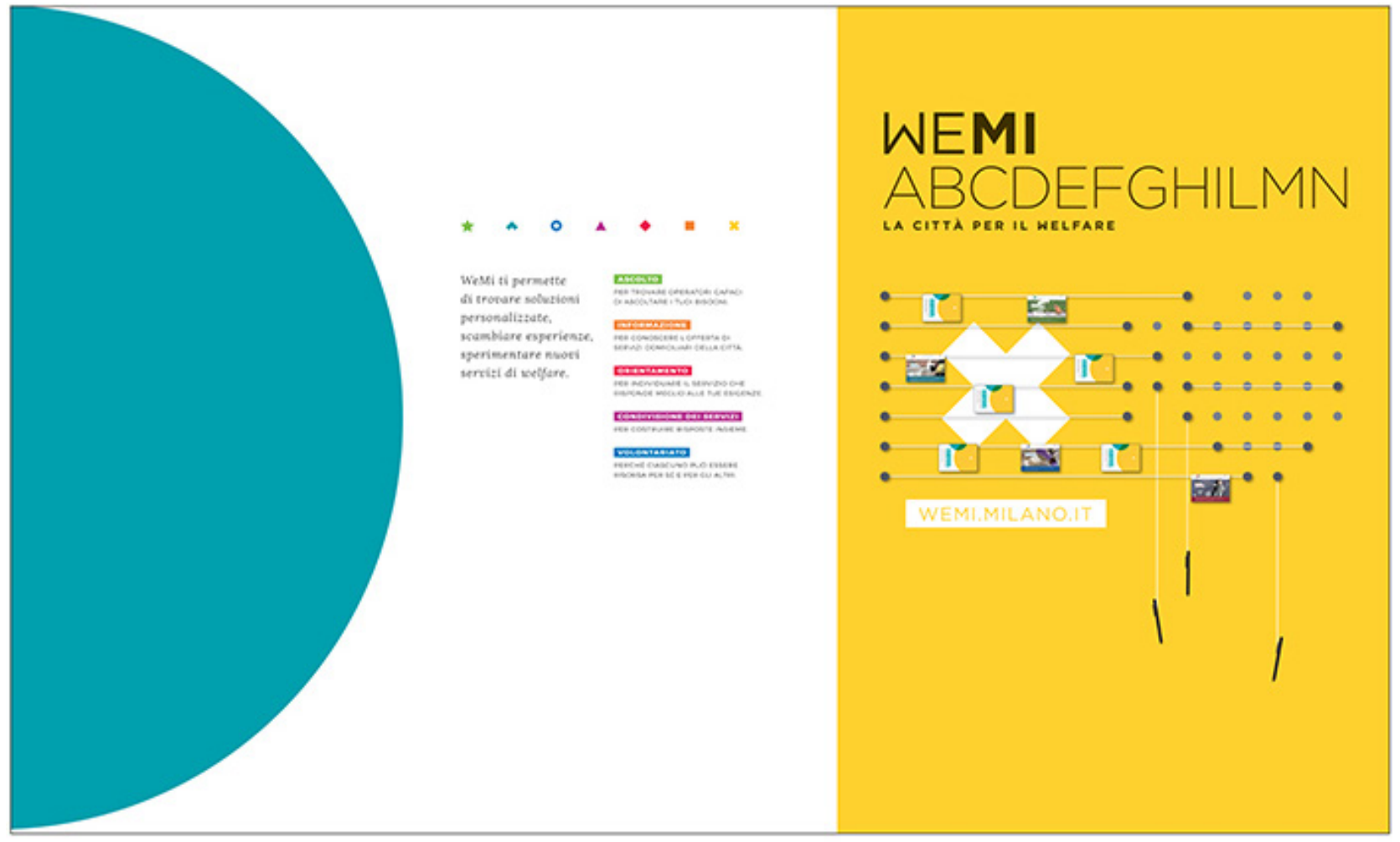

Fig. 11. Parete, esempio di impaginazione-tipo.

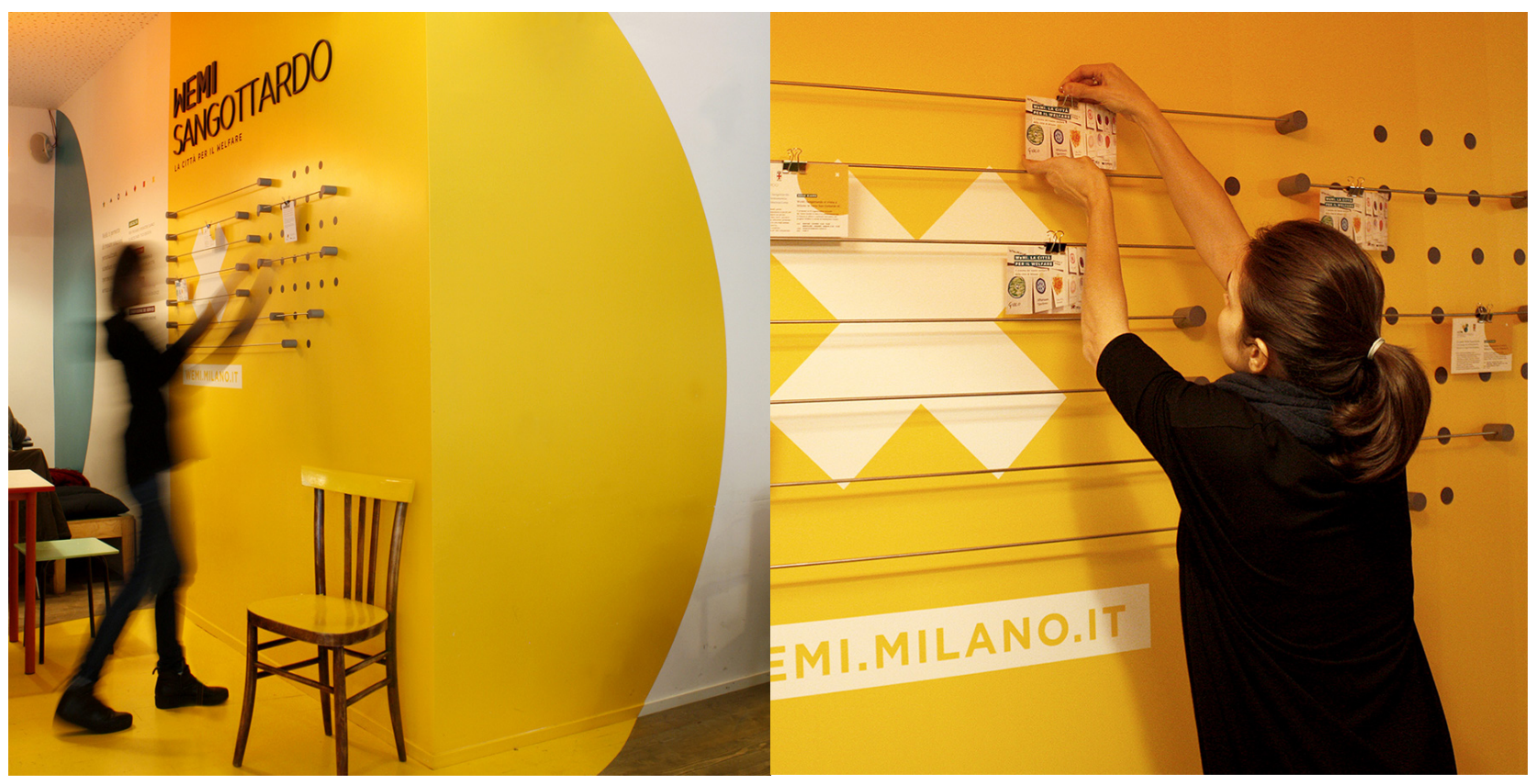

Fig. 12. Applicazioni del progetto di identità a scala architettonica. Archigrafie e dettagli di alcuni spazi WeMi. 


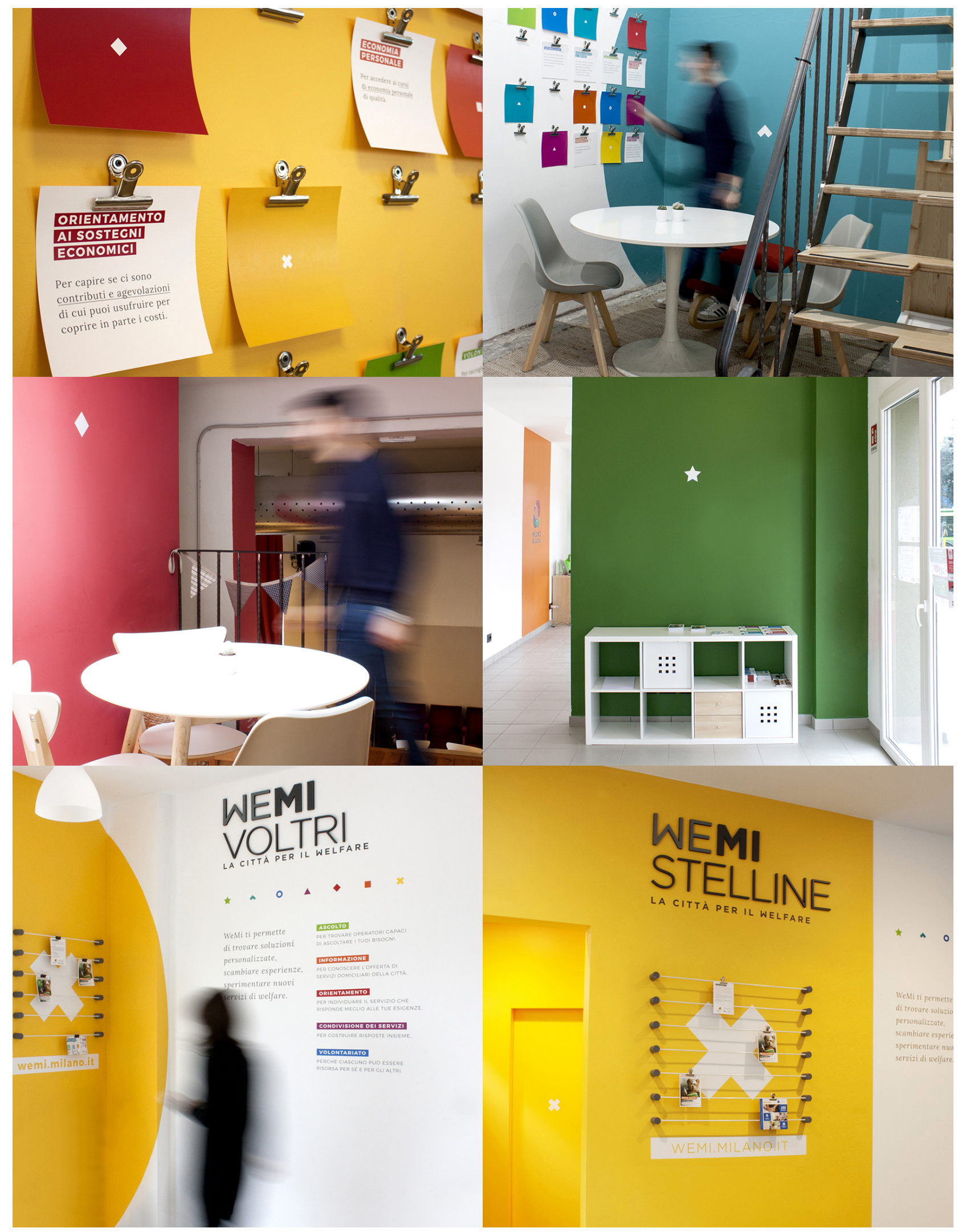

Fig. 13. Applicazioni del progetto di identità a scala architettonica. Archigrafie e dettagli di alcuni spazi WeMi. 


\section{Bibliografía}

Anceschi, G. (1981). Progettazione visiva: convenzioni e procedimenti di rappresentazioni. Budrio, Italia: Edizioni Officina Immagine.

Anceschi, G. (a cura di) (1984). Prima Biennale della Grafica. Milano, Italia: Arnaldo Mondadori Editore.

Anceschi, G. (1992). L'oggetto della raffigurazione. Milano, Italia: Etas.

Anceschi, G. (2011). Beyond Graphics. In G. Camuffo \& M.Dalla Mura (a cura di), Graphic design worlds/ words edited. Milano, Italia: Electa (La Triennale di Milano, Design Museum).

Baule, G. (2017). Rileggere la comunicazione di pubblica utilità. In V. Bucchetti (a cura di), Un'interfaccia per il welfare. Le funzioni sociali del design della comunicazione (pp.23-34). Milano, Italia: FrancoAngeli.

Baule, G., \& Caratti, E. (a cura di) (2016), Design è Traduzione. Il paradigma traduttivo per la cultura del progetto. "Design e Traduzione": un manifesto. Milano, Italia: FrancoAngeli.

Baule, G. (2005), Etiche della comunicazione. LineaGrafica 359, pp. 12-13.

Belpoliti, M., Canova, G. \& Chiodi S. (a cura di) (2007). Annisettanta, Milano, Italia: Skira.

Bollini, L. (2003). I "Manifesti", coscienza etica della professione. In L. Bollini \& C. Branzaglia (a cura di), No Brand more profit / Etica e comunicazione. Milano, Italia: Aiap Edizioni.

Brovelli, S., Farrauto, L., \& Sfligiotti S. (a cura di) (2012). Spazio Comune. Progetto grafico, n. 22, 2012.

Bucchetti, V. (2017). WeMi: un progetto di identità per il welfare. Territorio, n. 8, p. 99-105.

Bucchetti, V. (a cura di) (2017), Un'interfaccia per il welfare. Le funzioni sociali del design della comunicazione, Milano,Italia: FrancoAngeli.

Celaschi, F. (2016). Non industrial design. Contributi al discorso progettuale, Vignate, Italia: Luca Sossella Edizioni.

Consalez, L. (2017). La costruzione di uno spazio ibrido. Co-progettazione; elementi e limiti del progetto: RABWeMi SanGottardo, Territorio, pp. 88-98.

Mancini, P. (2002a). Manuale di comunicazione pubblica, Roma-Bari, Italia: Laterza.

Mancini, P. (2002b). Perché comunicazione pubblica? Le ragioni sociali di uno sviluppo impetuoso. Quaderni di sociologia, 30: 5-15.

Mondzain, M.J. (2015). L'image peut-elle tuer?. Montrouge, Francia: Bayard Editions.

Meroni, A. (2003). Rappresentare soluzioni: strumenti e tecniche per raccontare i servizi. In:

E. Manzini \& F. Jégou Quotidiano sostenibile. Scenari di vita urbana. Milano, Italia: Edizioni Ambiente.

Papanek, V. (1971). Design for the Real World: Human Ecology and Social Change, New York, USA: Pantheon Books.

Papanek, V. (1995), The Green Imperative. Singapore: Thames \& Hudson.

Papanek, V. (1983), Design for a Human Scale. New York, USA: Van Nostrand Reinhold Co.

Pignotti, L. (1984), Appunti pe runa grammatica e una identificazione della grafica di pubblica utilità. In G.Anceschi (a cura di), Prima Biennale della Grafica, Milano, Italia: Arnoldo Mondadori.

Rosina, A., \& Sorgi, S. (2016). Il futuro che (non) c'è. Milano, Italia: Università Bocconi Editore.

Steiner, A. (1973). La grafica degli Enti Pubblici. Linea grafica, 1, 2, 3.

Sinni, G. (2018). Una, nessuna, centomila. L'identità pubblica da logo a piattaforma. Macerata, Italia: Quodlibet.

Visconti, P. (2017). I sistemi di identità per il welfare. In V. Bucchetti (a cura di), Un'interfaccia per il welfare. Le funzioni sociali del design della comunicazione. Milano, Italia: FrancoAngeli. 